\title{
Phenobarbital analysis in biological matrix (blood) by high performance liquid chromatography (HPLC)
}

\author{
Ouakouak Hamza, Ben Mohamed Moktar, Ben Chohra Mostafa, \\ Abdelhamid Zeghdaoui* \\ Ecole Supérieure de Police-Chateauneuf, Laboratory of Scientific Police Cité El Ziania, \\ ben Aknoun Algiers, Algeria \\ *E-mail address: salah.neghmouche@gmail.com
}

\begin{abstract}
In this work, we have tried to contribute to the analysis of phenobarbital in the blood. To do this, we used different analytical techniques such as liquid chromatography (HPLC). The results obtained in the course of our work, we can conclude that: The HPLC method was separate phenobarbital endogenous blood components, and optimizing the conditions of chromatographic separation as the composition of the mobile phase consisting of $20 \%$ acetonitrile $+20 \%$ methanol + $60 \%$ ammonium acetate buffer. The extraction with diethyl ether to $\mathrm{pH}=4$; The chromatographic column, microbondapack ZORBAX C18. A system of diode-array UV detection at $254 \mathrm{~nm}$.
\end{abstract}

Keywords: Phenobarbital; HPLC; blood; extraction; biological matrix

\section{INTRODUCTION}

In the mid-1970s Barbiturates (BB) have been widely prescribed in developed countries as hypnotics or tranquilizers [1-2]. Phenobarbital held a respectable rank among the best selling drugs [3]. The duration of action makes them different classification barbiturates short ultra-short effects, intermediate or extended. During the last two decades Barbiturates have declined due to their replacement therapy by much safer benzodiazepines. They are however still used as an anesthetic in short duration of action and as anticonvulsants in the treatment of epilepsy [4].

This drug class plays longer a marginal role in forensic toxicology [5-6]. Forensic toxicology, toxic origin of death is usually determined by reference to the concentration of xenobiotic close of literature data and reference values for therapeutic [7-8], toxic and lethal concentrations. The objective of our work is to look at a topic that has absorbed phenobarbital, the concentration of the latter in the blood. For this purpose, we used analytical techniques developed enough such as HPLC coupled with a DBD.

The HPLC method was based on that used in the Laboratory of Scientific Police in Algiers for general research of organic toxic. However, it presents a major problem because of the interference of endogenous compounds with blood analyze. Changes have been made to remedy this, and get a good separation between phenobarbital and these compounds. 


\section{MATERIALS AND METHODS}

\section{1. The reagents and solvents}

Acetonitrile HPLC grade supplied by Panreac.

Methanol HPLC grade supplied by Merck.

Ammonium acetate supplied by Merck.

deionized water

\section{2. The standard solutions}

A mother solution of phenobarbital (provided by the Swiss Laboratory Dolder Ltd. /) were prepared in methanol at $1 \mathrm{mg} / \mathrm{ml}$ is $1 \mu \mathrm{g} / \mu \mathrm{l}$ is $1000 \mathrm{ng} /$ microl From the stock solution, solutions girls $200 \mathrm{ng} / \mu \mathrm{l}, 100 \mathrm{ng} / \mathrm{microl}, 50 \mathrm{ng} / \mathrm{microl}, 20 \mathrm{ng} / \mathrm{microl}, 10 \mathrm{ng} / \mathrm{microl}, 5 \mathrm{ng} /$ microl were prepared. They are kept at $10^{\circ} \mathrm{C}$ and protected from light.

\section{3. Apparatus and chromatographic conditions}

The equipment of high performance liquid chromatography coupled with a diode array detector (HPLC / DBD) (Agilent 1100series) used consists of the following elements:

- Automatic injector

- Detector UV / Vis diode-array

- ZORBAX SB C18 column (Agilent) (150 mm x $4.6 \mathrm{~mm}$ id, particle size 3.5 microns).

- Thermostatically controlled column oven, the temperature was set to $30{ }^{\circ} \mathrm{C}$.

\subsection{Optimization of the composition of the mobile phase}

To optimize the conditions for analysis of phenobarbital by HPLC method using a C18 column and a diode array detector, we tested different compositions of mobile phases.

\section{5. Mobile phase: acetonitrile ( $\mathrm{ACN} /$ ) phosphate buffer at $\mathbf{p H}=\mathbf{3 . 0 2}$}

Operating conditions:

- Mobile phase: phosphate buffer $0.02 \mathrm{M} \mathrm{KH}_{2} \mathrm{PO}_{4}$ at $\mathrm{pH}=3.02$ / $\mathrm{ACN}$ (see details in Table 2 gradient)

- Flow rate: $0.5 \mathrm{ml} / \mathrm{min}$

- Volume injected: 1 mu.1

- Analysis time: 15 min-Etalon Internal: Ethyl paraben in a solvent S1 to $30 \mathrm{mg} / 1$

- S1: Ultrapure water / ACN / acetic acid (70/26/4)

The elution of phenobarbital, using the mobile phase acetonitrile / phosphate buffer at different compositions yielded the results that are reported in Table 1 and The resulting chromatogram is shown in Figure 1.

Table 1. Detail of the gradient elution of the mobile phase ACN / phosphate buffer.

\begin{tabular}{|c|c|c|c|}
\hline time (mn) & \% ACN & \% buffer & Debits (ml/mn) \\
\hline 0 & 10 & 90 & 0.5 \\
\hline 10 & 55 & 45 & 0.5 \\
\hline 15 & 55 & 45 & 0.5 \\
\hline
\end{tabular}




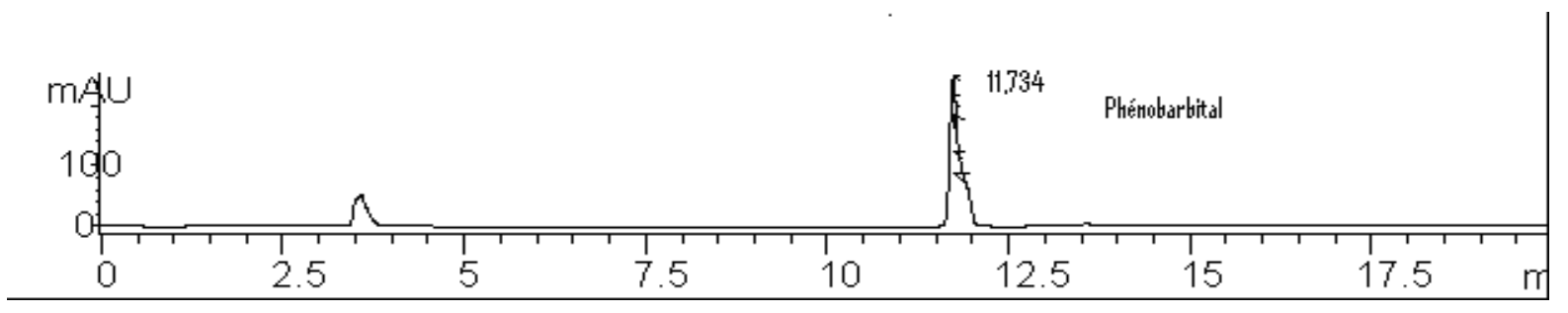

Figure 1. Chromatogram obtained with the ACN / phosphate buffer mobile phase pH $=3.02$.

Through the results, we note that retention times are long, sometimes exceeding 15 minutes for some compositions of the mobile phase. To optimize the analysis and resolution time, we changed the composition of the mobile phase, changing the nature of the buffer mixture and adding a polar solvent.

\section{6. Mobile phase: $\mathrm{ACN} / \mathrm{MetOH} /$ ammonium acetate buffer}

The method used at the forensic laboratory for research of toxic organic generally employs a mobile phase consisting of: Acetonitrile / methanol / ammonium acetate buffer $(30 / 30 / 40)(\mathrm{v} / \mathrm{v} / \mathrm{v})$

-Column: ZORBAX SB C18 (Agilent) (150mm x $4.6 \mathrm{~mm}$ id, particle size 3.5 microns).

- Flow rate: $1 \mathrm{ml} / \mathrm{min}$

- Injected Volume: $10 \mu 1$

- Detection: UV $254 \mathrm{~nm}$

Under these conditions we get a very good resolution of phenobarbital, the retention time is 2 minutes as shown in Figure 2.

However, in the biological matrix interferes with the peak of an endogenous compound plasma as shown in the chromatogram shown in Figure 3.

To eliminate the interference phenomena that may occur during the elution of phenobarbital, we acted on the composition of the mobile phase. For this, we have prepared a series of mobile phases consisting of acetonitrile buffer / methanol mixture / Ammonium Acetate in different compositions. 


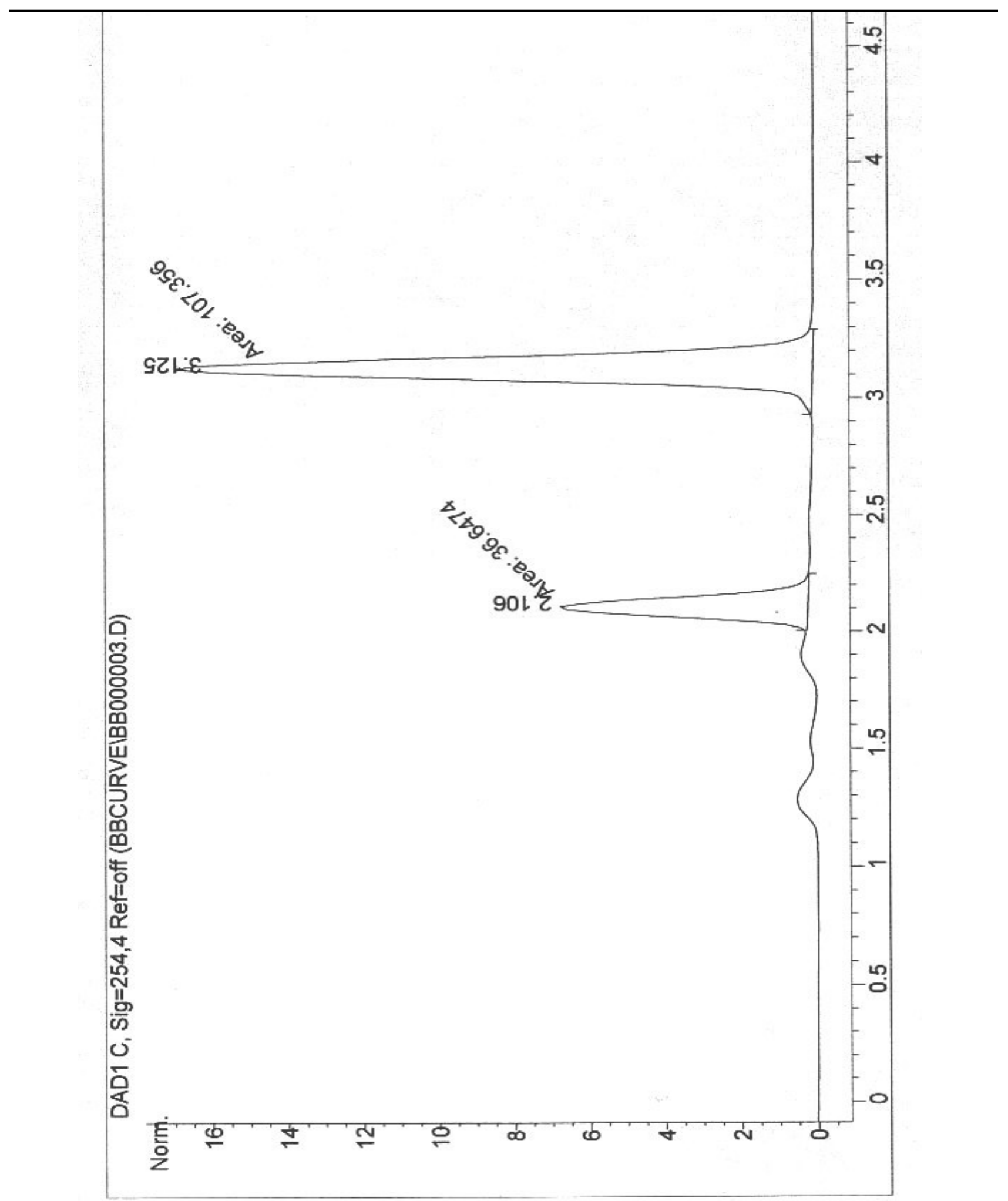

Figure 2. Chromatogram in methanol solution with pentobarbital $(\mathrm{r}=3.125 \mathrm{~min})$ as SI. 


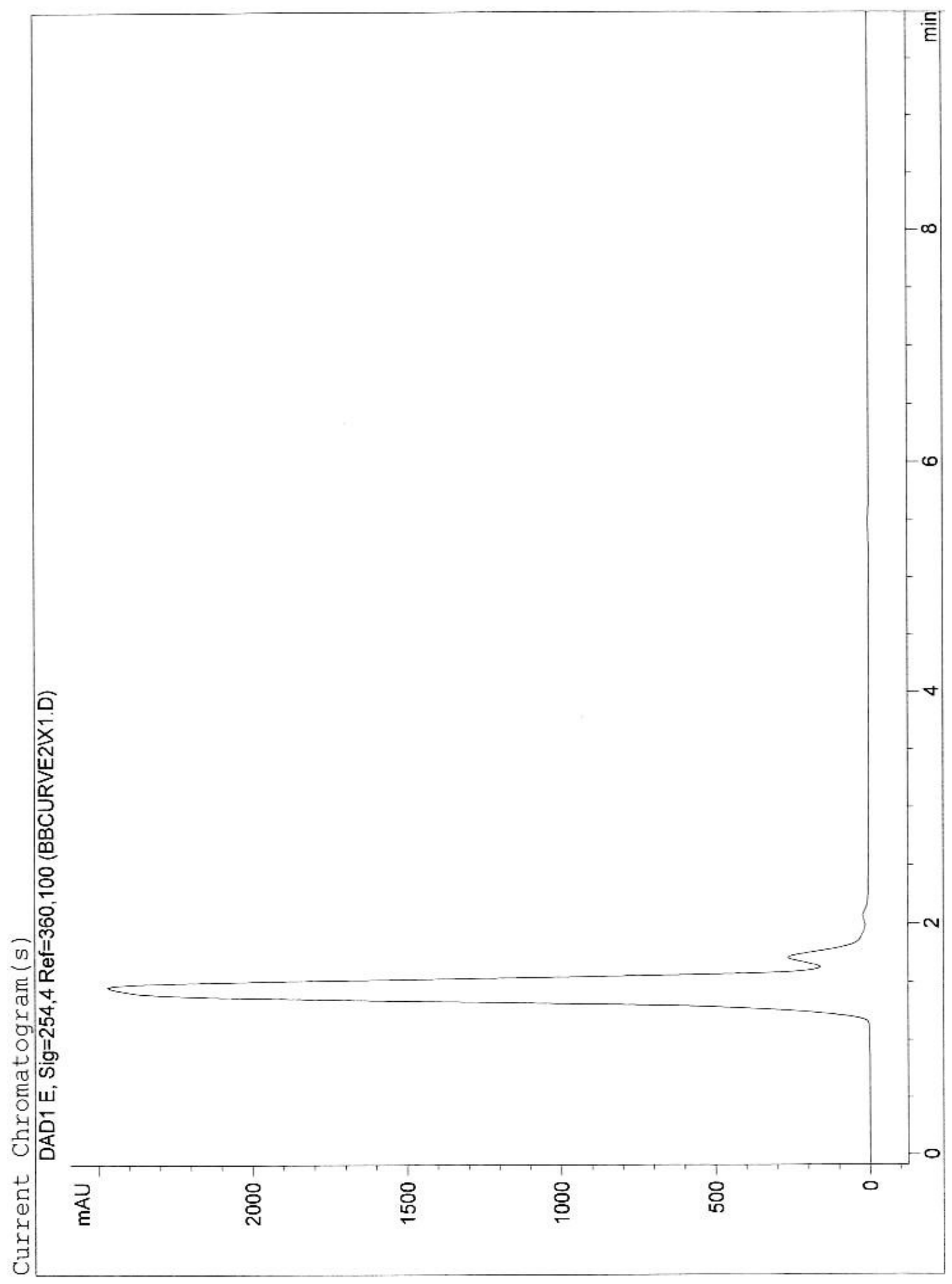

Figure 3. Chromatogram obtained with a white blood exhibits peaks (neighbor tr 2, min 0) which may interfere with that of phenobarbital. 


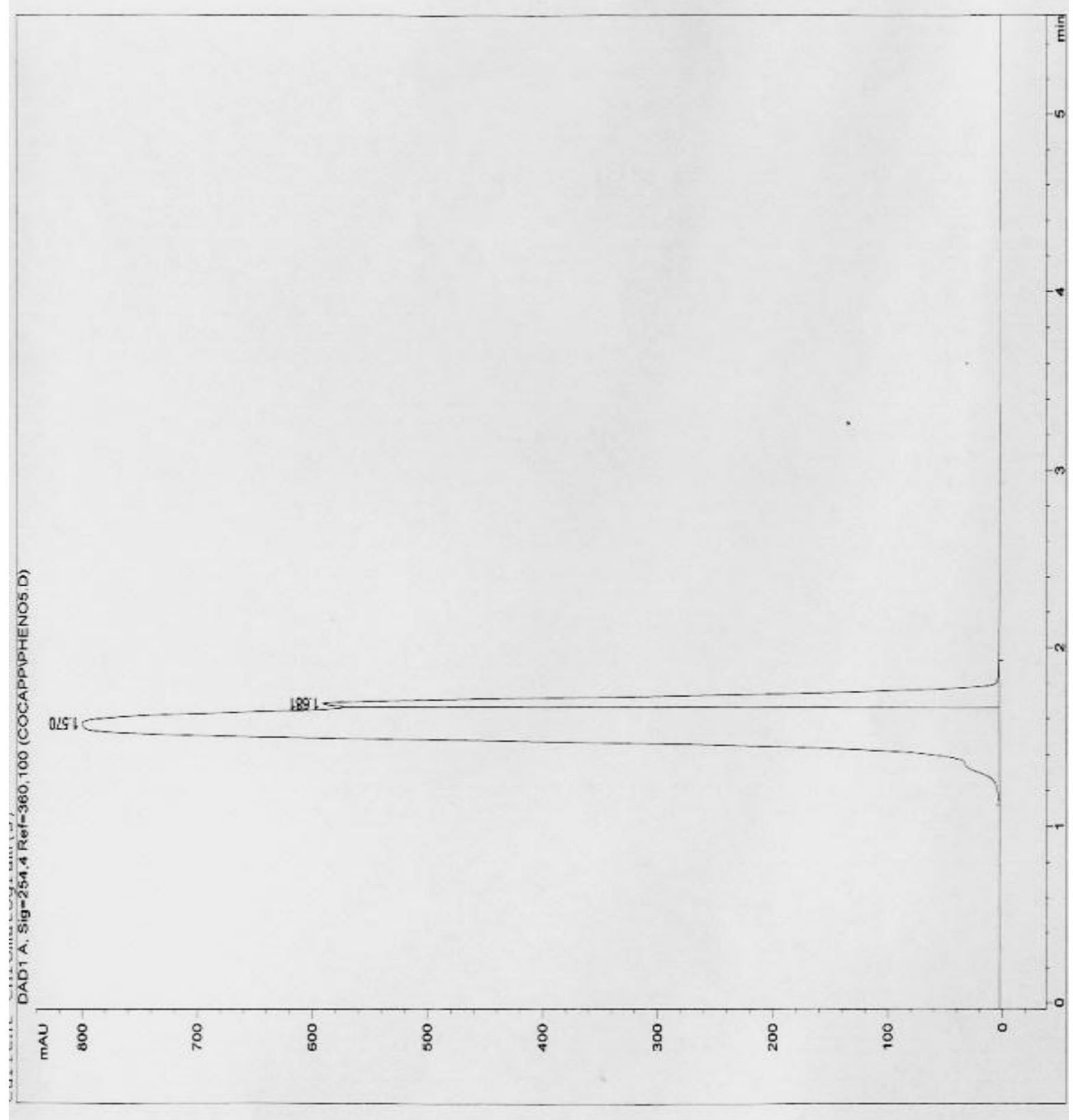

Figure 4. Chromatogram obtained with the composition 70/10/20.

Table 2 lists all of the various compositions tested mobile phase, and the retention times obtained phenobarbital. 
Table 2. retention time corresponding to the various compositions of the mobile phase.

\begin{tabular}{|c|c|}
\hline Phase composition (Ac/Met/ buffer) & tr (min) \\
\hline $70 / 10 / 20$ & 1,57 \\
\hline $30 / 30 / 40$ & 2,3 \\
\hline $30 / 10 / 60$ & 2,8 \\
\hline $40 / 20 / 40$ & 2,121 \\
\hline $40 / 10 / 50$ & 2,81 \\
\hline $\mathbf{2 0 / 2 0 / 6 0}$ & $\mathbf{4 , 1 3}$ \\
\hline
\end{tabular}

\section{RESULTS AND DISCUSSION}

Table 3. Values of the resolution based on the percentage of acetonitrile (ACN).

\begin{tabular}{|c|c|c|c|c|}
\hline $\mathbf{R}$ & 5 & 0,6 & 0,2 & 0.34 \\
\hline \% ACN & 20 & 30 & 40 & 70 \\
\hline
\end{tabular}

Figure 5 is a graphical representation of the resolution based on the percentage of acetonitrile (ACN).

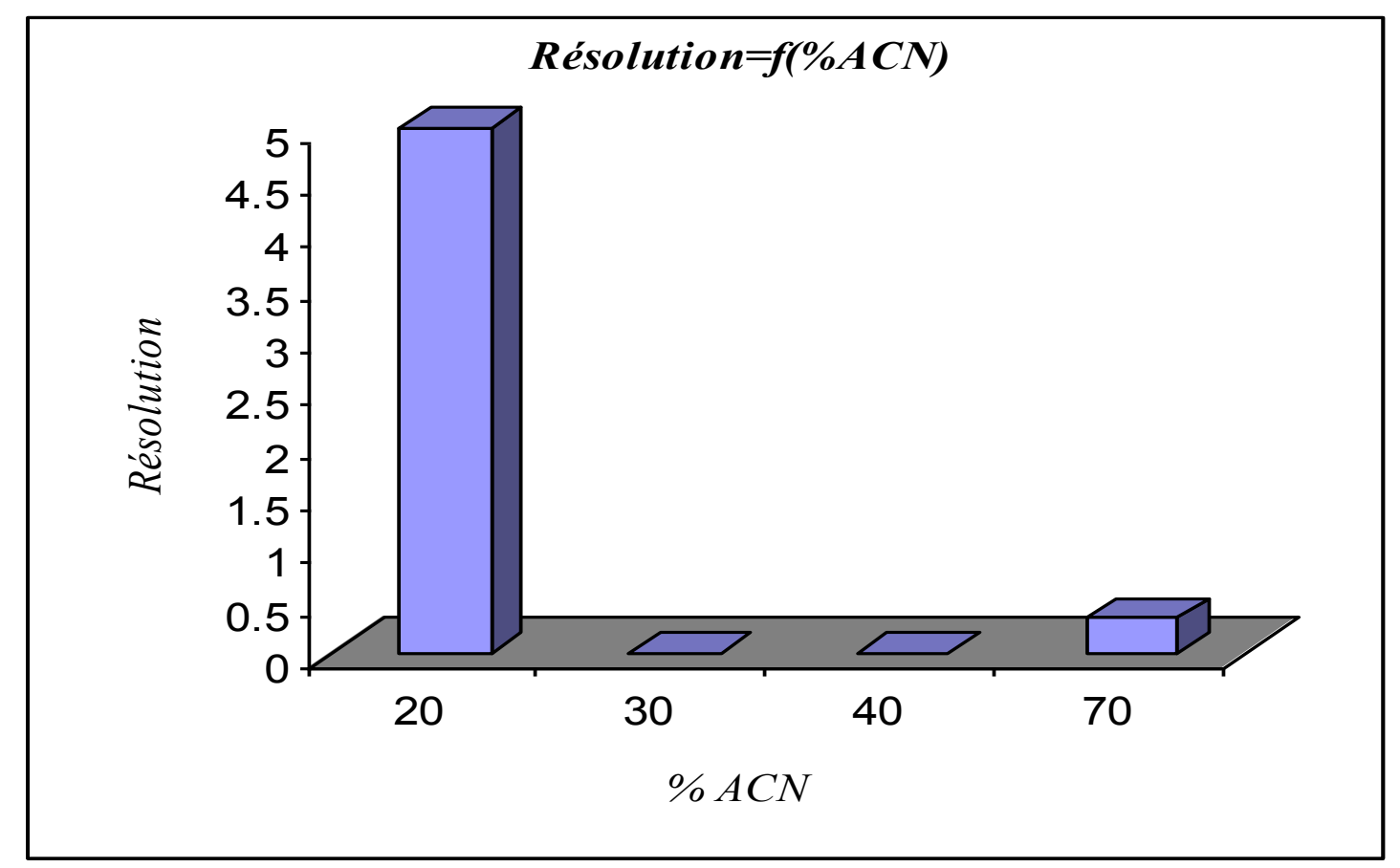

Figure 5. Resolution on the percentage of CAN. 
We determined the peak resolutions between phenobarbital and the peak of endogenous compound for all mobile phases studied. The route curves giving the resolution (R) based on the percentage of acetonitrile, methanol, and acetate buffer in the mobile phase is represented by histograms component Figures 5, 6 and 7 respectively.

Table No. 3 contains the values of the resolution obtained for the different percentages of acetonitrile (ACN).

Table No. 4 shows the variation of the resolution based on the percentage of ammonium acetate buffer content in the mobile phase

Table 4. Changes the resolution based on the percentage of ammonium acetate buffer.

\begin{tabular}{|c|c|c|c|c|}
\hline $\mathbf{R}$ & 0,34 & 0,40 & 0,2 & 5 \\
\hline \% Tampon & 20 & 40 & 50 & 60 \\
\hline
\end{tabular}

Figure 6 shows the resolution based on the percentage of ammonium acetate buffer.

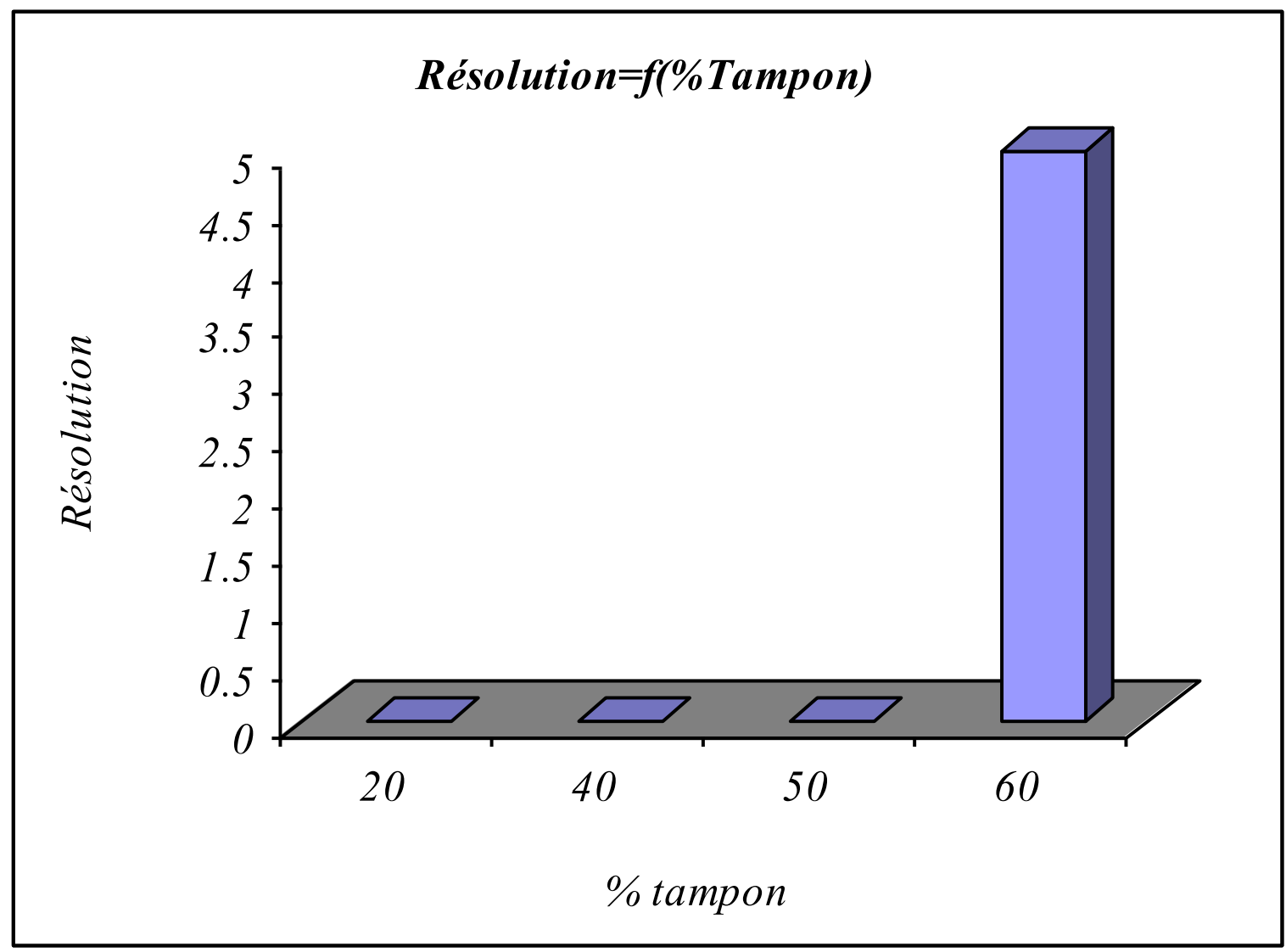

Figure 6. Change the resolution based on the percentage of acetate buffer solution. 
Table No. 5 contains the values of the resolution calculated according to the percentage of methanol in the mobile phase.

Table 5. Changes the resolution based on the percentage of methanol.

\begin{tabular}{|c|c|c|c|}
\hline $\mathbf{R}$ & 0,63 & 5,0 & 0,48 \\
\hline \% Méthanol & 10 & 20 & 30 \\
\hline
\end{tabular}

Figure 7 shows the variation of the resolution based on the percentage of methanol.

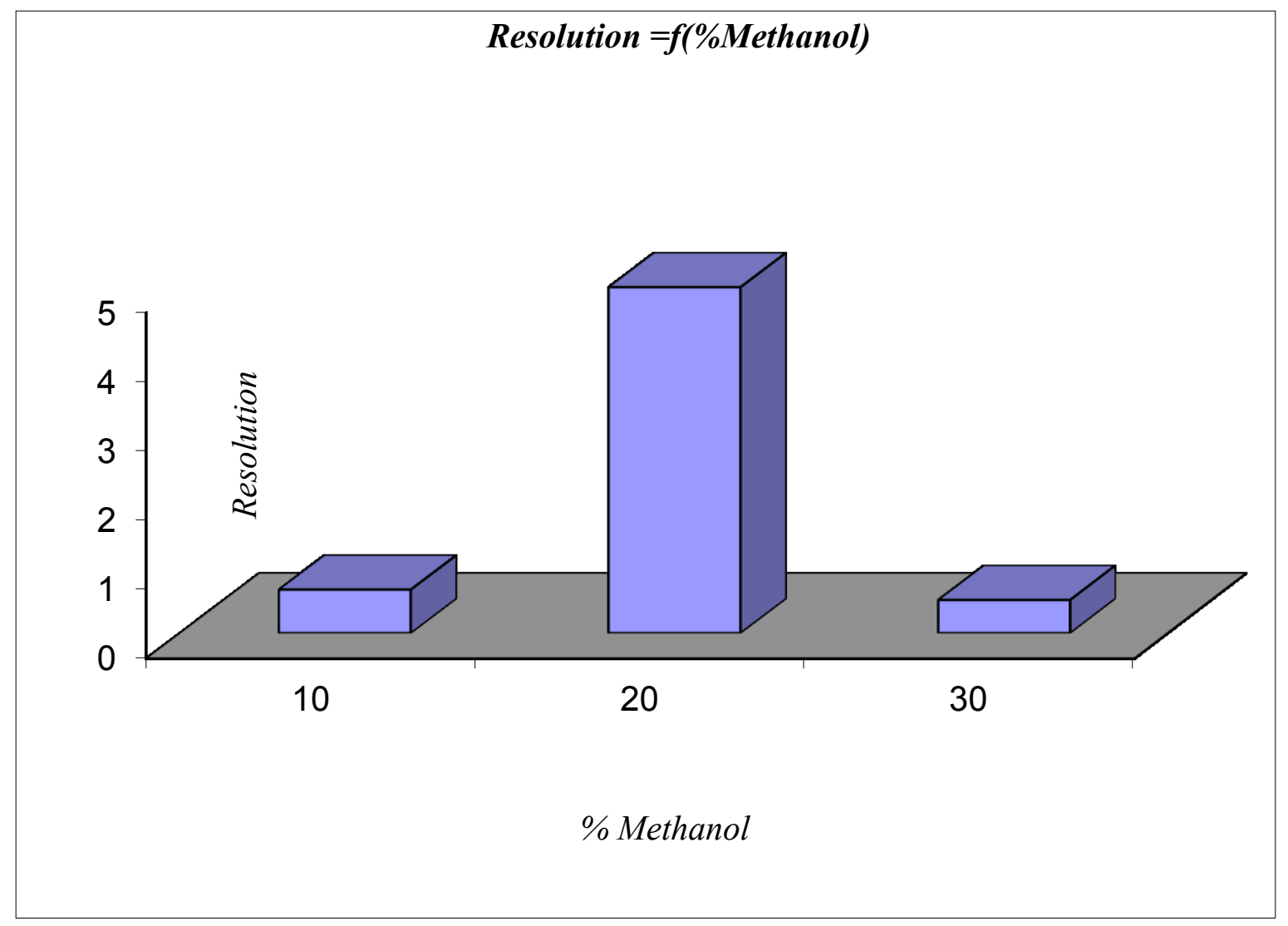

Figure 7. Resolution on the percentage of methanol.

We analyzed, organic toxic, phenobarbital, in the blood. It is known that for performing the assay of an analyze in a biological medium, preparation of the sample, which comprises extracting the compound of interest is required. Liquid liquid extraction (LLE) and solid phase extraction (SPE) methods are all set for the preparation of samples from a biological medium.

In our work we opted for liquid liquid extraction (LLE). Several mixtures of extraction solvents were tested. We give below the principle of the method and parameters that can influence the performance of the extraction 


\section{CONCLUSION}

In this work, we have tried to contribute to the analysis of phenobarbital in the blood. To do this, we used different analytical techniques such as liquid chromatography (HPLC). The results obtained in the course of our work, we can conclude that: The HPLC method was separate phenobarbital endogenous blood components, and optimizing the conditions of chromatographic separation as The composition of the mobile phase consisting of $20 \%$ acetonitrile $+20 \%$ methanol $+60 \%$ ammonium acetate buffer. The extraction with diethyl ether to $\mathrm{pH}=4$; The chromatographic column, microbondapack ZORBAX C18. A system of diode-array UV detection at $254 \mathrm{~nm}$.

\section{Reference}

[1] Blackwell B., JAMA 225 (1973) 1637-1641.

[2] Y. Kouno, C. Ishikura, M. Homma, K. Oka, J. Chromatogr. 622 (1993) 47-52.

[3] M. Moriyama, H. Domoto, S. Yamashita, K. Furuno, R. Oishi, H. Kawasaki, Y. Gomita, Acta Med. Okayama 49 (1995) 237.

[4] M. Moriyama, S. Yamashita, K. Furuno, T. Sato, H. Domoto, Y. Yamotogi, H. Kawasaki, Y. Gomita, Jpn. J. Pharmacol. 73 (1997) 191.

[5] R. Liu, A. Mckeehan, B. Cinnamon, G. Foster, W. Bensley, J. Langner, A. Walia, J. Forensic Sci. 39 (1994) 1504.

[6] H. Nau, D. Rating, I. Hauser, S. Koch, H. Helge, Eur. J. Clin. Pharmacol. 18 (1980) 31.

[7] Neghmouche N. S., Khelef A., Lanez T., J. Fund. App. Sci. 1(1) (2009) 23-30.

[8] H. Levert P. Odou, H. Robert, Biomed Chromatogr. 16 (2002) 19-24. 